\title{
REVAMMAD JMO Special Edition
}

This special edition of the Journal of Modeling in Ophthalmology features papers from the Early Career Researchers of the EU Marie Cutie Integrated Training Network project REVAMMAD (Retinal Vascular Modeling, Measurement and Diagnosis), which ran from April 2013 to March 2017. The REVAMMAD project, recognizing the potential complementarity between computational modeling, data analysis, theoretical understanding, and clinical practice, was designed to train a cadre of early stage researchers (ESRs) able to explore various interactions between these themes in the context of retinal pathology. The ESRs each undertook a different interdisciplinary project, underpinned by a shared training program, placements at different member institutions, and supervisory input from multiple sites. The project led to a number of scientific contributions, from which this special edition draws together some illustrative examples. Each one touches upon a different aspect of the interaction between modeling, measurement by image analysis, and theoretical analysis.

In Calivá et al. we see features derived from computational flow modeling being analyzed for correlation with the progression of diabetic retinopathy (DR). The literature contains a number of studies showing that measurable visual features of the retina correlate with DR progression (e.g., vessel caliber); this paper is the first attempt to move the analysis from the directly measurable to the hemodynamically significant.

Annunziata et al. investigate the correlation of hemodynamic parameters with vessel tortuosity, utilizing data from a mesenteric vascular network - tortuosity being a well-known visual sign of various retinal pathologies - with a view to better understanding the underlying hemodynamic issues. The study indicates that tortuosity is related to multiple hemodynamic parameters.

Hernandez-Matas et al. introduce a dataset providing ground truth data for image registration. Many problems of interest in retinal analysis manifest in changes to the retina, the detection of which requires image registration prior to change detection. The inclusion of image pairs with ground truth reference point correspondences facilitates the development and comparison of registration algorithms.

Wigdahl et al. address the problem of optic nerve head (ONH) localization in images by modeling flow in a different way: a shortest path algorithm run across the retinal area at various test points naturally tends to route through the $\mathrm{ONH}$ (where blood vessels converge), providing a simple and effective method to enhance the image for $\mathrm{ONH}$ detection. The result is an efficient, effective, and novel approach. 
Neganova introduces the first approach to modeling the short-term myogenic response of vessel wall cells and connective tissue to a pressure stimulus. The modeling suggests that the myogenic response is important in regulating blood flow under pressure variations, achieving more stable flow than a non-myogenic alternative model, and working holistically in concert with the naturally occurring asymmetry of the vascular network.

Kotsiliti et al. provides a quite different type of model, utilizing machine learning to predict DR from clinical patient data rather than from retinal images. The model suggests that a combination of standard patient information and routine biochemical tests gathered consistently over a screening period provides a strong correlation with disease progression.

Overall, the papers illustrate the broad opportunities for scientific discovery that emerge when clinicians, theorists, and computational analysts cooperate. The REVAMMAD project has been enriching in multiple directions, with modeling concepts leading to novel approaches to image analysis, analysis providing new insights and more accurate data for modelers, and clinical insights guiding the development of techniques. Underlying all, cooperation in generating data has been essential. This special edition can only touch upon selected aspects of the range of work undertaken in the project; it has been an honor to help introduce an enthusiastic cohort of young scientists to this fascinating discipline and to see the wealth of new ideas and insights they have brought to bear.

Professor Andrew Hunter

University of Lincoln

Dr. Jens Christian Brings Jacobsen

University of Copenhagen 